\title{
The optimal period of Ca-EDTA treatment for parthenogenetic activation of porcine oocytes during maturation culture
}

\author{
Yasuhiro MORITA ${ }^{1)}$, Masayasu TANIGUCHI ${ }^{1)}$, Fuminori TANIHARA ${ }^{2)}$, Aya ITO ${ }^{1)}$, Zhao NAMULA ${ }^{1)}$, \\ Lanh Thi Kim DO ${ }^{1)}$, Mitsuhiro TAKAGI ${ }^{1)}$, Tatsuya TAKEMOTO ${ }^{3)}$ and Takeshige OTOI $^{2) *}$ \\ ${ }^{1)}$ The United Graduate School of Veterinary Science, Yamaguchi University, Yamaguchi 753-8515, Japan \\ ${ }^{2)}$ Faculty of Bioscience and Bioindustry, Tokushima University, Tokushima 770-8501, Japan \\ 3)Division of Embryology, Fujii Memorial Institute of Medical Sciences, Tokushima University, Tokushima 770-8503, Japan
}

(Received 15 November 2015/Accepted 22 February 2016/Published online in J-STAGE 4 March 2016)

ABSTRACT. The changes triggered by sperm-induced activation of oocytes, which are required for normal oocyte development, can be mediated by other agents, thereby inducing the parthenogenesis. In this study, we exposed porcine oocytes to 1 mM Ca-EDTA, a metal-ion chelator, at various intervals during $48 \mathrm{hr}$ of in vitro maturation to determine the optimum period of Ca-EDTA treatment for parthenogenetic activation. When the oocytes were cultured with or without Ca-EDTA from $36 \mathrm{hr}$ (post-12), $24 \mathrm{hr}$ (post-24), $12 \mathrm{hr}$ (post-36) and $0 \mathrm{hr}$ (post-48) after the start of maturation culture, the blastocyst formation rates were significantly higher $(P<0.05)$ in the post-24, post-36 and post-48 groups (3.3\%, $4.0 \%$ and $2.6 \%$, respectively) than those in the control group without treatment $(0 \%)$. Furthermore, when the oocytes were cultured with Ca-EDTA for $0 \mathrm{hr}$ (control), $12 \mathrm{hr}$ (pre-12), $24 \mathrm{hr}$ (pre-24), $36 \mathrm{hr}$ (pre-36) and $48 \mathrm{hr}$ (pre-48) from the start of maturation culture, the oocytes formed blastocysts only in the pre-36 and pre- 48 groups $(0.4 \%$ or $0.8 \%$, respectively). Pronuclei $(<66.7 \%)$ were observed only when the periods of Ca-EDTA treatment were more than $12 \mathrm{hr}$ during maturation culture. In the control group, no pronuclei were detected. Our findings demonstrate that porcine immature oocytes can be parthenogenetically activated by Ca-EDTA treatment for at least $24 \mathrm{hr}$ to $36 \mathrm{hr}$ during maturation culture, leading to pronucleus formation followed by the formation of blastocysts.

KEY WORDS: blastocyst, Ca-EDTA, nuclear status, parthenogenesis, porcine oocyte

doi: 10.1292/jvms.15-0658; J. Vet. Med. Sci. 78(6): 1019-1023, 2016

Mammalian oocytes are arrested at metaphase II (MII) of meiosis and are only released from this stage when an activation signal is naturally provided by the entry of sperm $[18,26]$. Sperm-induced egg activation triggers a series of events, such as induction of intracellular $\mathrm{Ca}^{2+}$ oscillations that reduce the activity of the maturation promoting factor (MPF) and enable resumption of meiosis [7]; this is followed by the formation of male and female pronuclei (PN) [1]. These events are indispensable for normal oocyte development [8].

Artificially, oocyte activation can be induced by stimuli, such as ethanol [16, 17], calcium ionophore A23187 [25], ionomycin [5], electroporation [6], $\mathrm{CaCl}_{2}$ [14], protein kinase inhibitors [15], G protein [14], cycloheximide [19], and strontium and barium ions [20], mimicking normal fertilization [21]. These reagents replace the natural activation signal of sperm [22] and induce the process of parthenogenesis, which is essential for the success of nuclear cloning and reprogramming of the donor nucleus. In pigs, parthenogenotes are also used for embryo transfer to promote pregnancy and piglet production. It has been demonstrated that a normal piglet was delivered by the co-transfer of in vitro produced

*Correspondence to: Otol, T., Faculty of Bioscience and Bioindustry, Tokushima University, Tokushima 770-8501, Japan. e-mail: otoi@tokushima-u.ac.jp

(C)2016 The Japanese Society of Veterinary Science

This is an open-access article distributed under the terms of the Creative Commons Attribution Non-Commercial No Derivatives (by-nc-nd) License $<$ http://creativecommons.org/licenses/by-nc-nd/4.0/>. embryos with parthenogenotes produced by electrical activation [9]. Parthenogenotes usually die by the 30th day of pregnancy, therefore useful to establish and maintain pregnancy in place of normal embryos.

Ethylenediamine tetraacetic acid (EDTA) saturated with $\mathrm{Na}^{+}$(Na-EDTA) has been successfully used for parthenogenetic activation of porcine immature oocytes at the germinal vesicle $(\mathrm{GV})$ stage, thereby enabling blastocyst formation from immature oocytes. In this case, PN formation starts directly from interphase, between the germinal vesicle breakdown (GVBD) and anaphase I/telophase I (AT) stages, and not via the MII stage. [3, 4]. Ca-EDTA at a concentration of $1 \mathrm{mM}$ has also been shown to induce PN formation from porcine and bovine $\mathrm{GV}$-stage oocytes after $48 \mathrm{hr}$ of maturation culture; however, mouse oocytes were not activated by this treatment [4]. Moreover, PN formation in bovine oocytes required Ca-EDTA treatment for 36-48 hr during the culture period. These studies indicate that EDTA treatment during maturation culture can induce the precocious formation of a pronucleus followed by the development of blastocysts by passing over the second meiotic maturation period. However, at present, little information is available regarding the parthenogenetic activation conditions required during Ca-EDTA treatment for the development of porcine oocytes.

To establish a new method of chemical activation, we examined the optimal period of Ca-EDTA treatment during maturation culture for enabling the parthenogenetic development of porcine oocytes to the blastocyst stage. 


\section{MATERIALS AND METHODS}

Collection and in vitro maturation (IVM) of oocytes: Porcine ovaries were obtained from prepubertal cross-bred gilts (Landrace, Large White and Duroc breeds) at a slaughterhouse and transported to the laboratory in $0.9 \%$ physiological saline at $35^{\circ} \mathrm{C}$ within $3 \mathrm{hr}$ of slaughter. Cumulus-oocyte complexes (COCs) were aspirated from follicles measuring 3 to $6 \mathrm{~mm}$ in diameter by using an 18-gauge needle connected to a $5-\mathrm{m} l$ disposable syringe and collected in modified phosphate-buffered saline (m-PBS; Nihonzenyaku, Fukushima, Japan) supplemented with $100 \mathrm{IU} / \mathrm{m} l$ penicillin $\mathrm{G}$ potassium (Meiji, Tokyo, Japan) and $0.1 \mathrm{mg} / \mathrm{m} l$ streptomycin sulphate (Meiji). Only COCs with a uniform ooplasm and a compact cumulus cell mass were used in this study. COCs were matured in maturation medium (IVM medium), as described by Azuma et al. [3] with minor modifications. Briefly, IVM medium consisted of tissue culture medium (TCM) 199 (Earle's salts) with 25 mM HEPES buffer (TCM 199; Life Technologies, Carlsbad, CA, U.S.A.) supplemented with $0.1 \%$ polyvinyl alcohol (PVA; Sigma-Aldrich, St. Louis, MO, U.S.A.), 3.05 mM D-glucose (Wako, Osaka, Japan), 0.91 $\mathrm{mM}$ sodium pyruvate (Life Technologies), $10 \mathrm{IU} / \mathrm{m} l$ equine chorionic gonadotropin (Kawasaki-Mitaka K.K., Kawasaki, Japan), $10 \mathrm{IU} / \mathrm{m} l$ human chorionic gonadotropin (KawasakiMitaka) and $50 \mu \mathrm{g} / \mathrm{m} l$ gentamicin (Sigma-Aldrich). COCs were treated with $1 \mathrm{mM}$ Ca-EDTA (Wako) according to the treatment schedules shown in Fig. 1. Oocytes were matured totally for $48 \mathrm{hr}$ in IVM medium including term incubated in IVM medium with $1 \mathrm{mM}$ Ca-EDTA. Approximately 10 COCs were cultured in each 100- $\mu$ l drop of IVM medium covered with a layer of mineral oil (Sigma-Aldrich) in a 35 $\times 10 \mathrm{~mm}$ petri dish (Becton Dickinson, Franklin Lakes, NJ, U.S.A.). All cultures were performed at $38.5^{\circ} \mathrm{C}$ in a humidified incubator containing $5 \% \mathrm{CO}_{2}$.

Embryo culture: After IVM culture, all oocytes were mechanically denuded of cumulus cells in TCM 199 supplemented with $0.1 \%(\mathrm{w} / \mathrm{v})$ hyaluronidase (Sigma-Aldrich) and cultured in North Carolina State University (NCSU) 23 medium [23] supplemented with $4 \mathrm{mg} / \mathrm{m} l$ bovine serum albumin (BSA; Sigma-Aldrich) and $50 \mu \mathrm{g} / \mathrm{ml}$ gentamicin in an atmosphere containing $5 \% \mathrm{CO}_{2}$ at $38.5^{\circ} \mathrm{C}$. After $72 \mathrm{hr}$, the embryos were transferred to fresh NCSU 23 medium supplemented with $4 \mathrm{mg} / \mathrm{ml}$ BSA (Sigma-Aldrich), $5.55 \mathrm{mM}$ d-glucose and $50 \mu \mathrm{g} / \mathrm{m} l$ gentamicin, and cultured for additional 5 days. The rates of cleavage and blastocyst formation were examined on day 3 and day 8 after in vitro culture, respectively.

Assessment of nuclear status: To evaluate the nuclear status of porcine oocytes after $48 \mathrm{hr}$ of IVM culture, oocytes were denuded of cumulus cells by repeated pipetting, mounted on slides, fixed in acetic acid : ethanol (1:3) for 2 days, stained with $1 \%(\mathrm{w} / \mathrm{v})$ orcein (Sigma-Aldrich) in $45 \%(\mathrm{v} / \mathrm{v})$ acetic acid (Wako) and examined under a phase contrast microscope.

Assessment of embryo development: On day 8 of culture, all embryos were fixed and permeabilized in PBS containing $3.7 \%(\mathrm{w} / \mathrm{v})$ paraformaldehyde and $1 \%(\mathrm{v} / \mathrm{v})$ Triton X-100
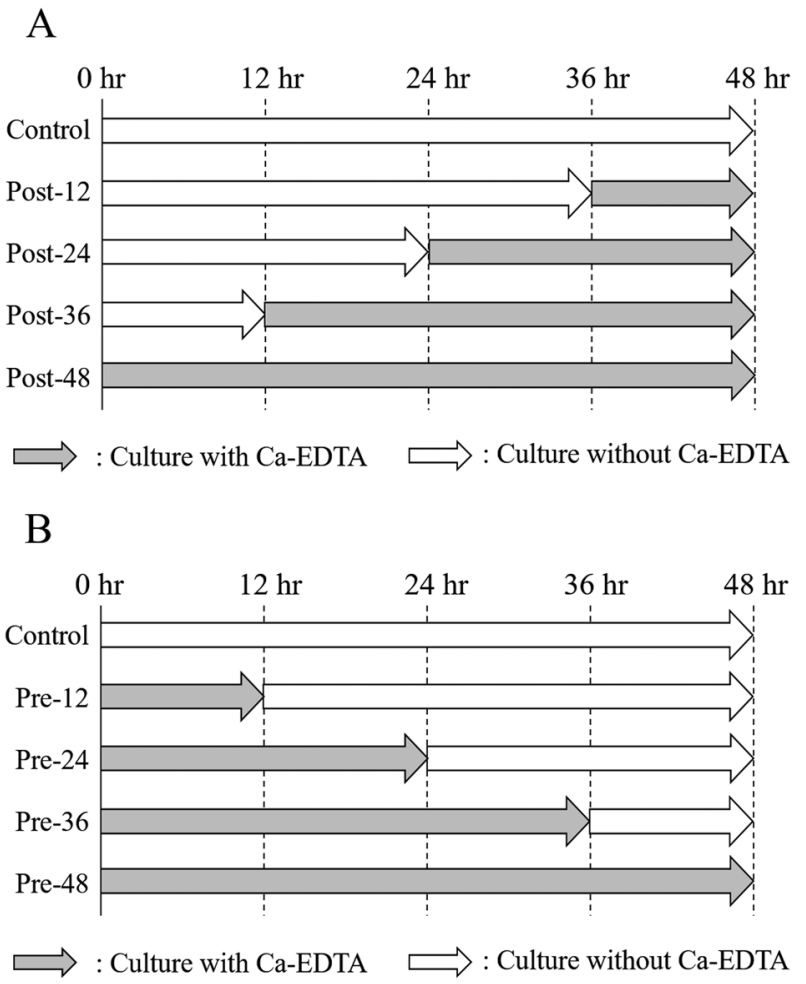

Fig. 1. Experimental design representing the schedules of CaEDTA exposure to porcine oocytes during maturation culture. A: treatment groups for pre-treatment experiment (Experiment 1); B: treatment groups for post-treatment experiment (Experiment 2 ). Filled and open arrows indicate culture with and without $\mathrm{Ca}$ EDTA, respectively.

(Sigma-Aldrich) at room temperature for $15 \mathrm{~min}$ and incubated in PBS containing $0.3 \%(\mathrm{w} / \mathrm{v})$ polyvinylpyrrolidone at room temperature for another $15 \mathrm{~min}$. Embryos were placed in a drop of mounting medium consisting of $90 \%$ (v/v) glycerol containing $1.9 \mu \mathrm{M}$ Hoechst 33342 (Sigma-Aldrich) on a slide, overlaid with a cover slip supported by four droplets of vaseline/paraffin, incubated overnight at $4^{\circ} \mathrm{C}$ and examined under a fluorescence microscope with a $355-\mathrm{nm}$ wavelength excitation filter. Embryos with a clear blastocoele were defined as blastocysts. The numbers of cleaved embryos and blastocysts were recorded.

Experimental design: In experiment 1, the induction of parthenogenetic development of porcine oocytes by posttreatment with Ca-EDTA during maturation culture was examined. COCs were randomly allocated to one of five treatment groups (Fig. 1A), cultured in IVM medium without Ca-EDTA for $48 \mathrm{hr}, 36 \mathrm{hr}, 24 \mathrm{hr}, 12 \mathrm{hr}$ and $0 \mathrm{hr}$ and subsequently incubated in IVM medium supplemented with $1 \mathrm{mM}$ Ca-EDTA for $0 \mathrm{hr}$ (control), $12 \mathrm{hr}$ (post-12), $24 \mathrm{hr}$ (post-24), $36 \mathrm{hr}$ (post-36) and $48 \mathrm{hr}$ (post-48). After $48 \mathrm{hr}$ of IVM culture, some oocytes were fixed and stained to examine the nuclear status of oocytes treated with Ca-EDTA. Remaining oocytes were cultured in NCSU 23 medium for 8 days and examined the embryo development as described 
above.

In experiment 2, the development of porcine oocytes by pre-treatment with Ca-EDTA during maturation culture was examined. COCs were incubated in IVM medium supplemented with $1 \mathrm{mM}$ Ca-EDTA for $0 \mathrm{hr}$ (control), $12 \mathrm{hr}$ (pre12), $24 \mathrm{hr}$ (pre-24), $36 \mathrm{hr}$ (pre-36) and $48 \mathrm{hr}$ (pre-48), and subsequently cultured in IVM medium without Ca-EDTA (Fig. 1B). After $48 \mathrm{hr}$ of IVM culture, some oocytes were fixed and stained to examine the nuclear status of oocytes treated with Ca-EDTA. Remaining oocytes were cultured in NCSU 23 medium for 8 days.

Statistical analysis: Experiments were repeated four times per treatment. The percentages of cleaved embryos and embryos developed to the blastocyst stage were subjected to arcsine transformation before analysis of variance (ANOVA). The transformed data were tested by ANOVA, followed by protected Fisher's least significant difference (LSD) test, using the StatView software (Abacus Concepts, Berkeley, CA, U.S.A.). Differences with a probability value of $P<0.05$ were considered statistically significant.

\section{RESULTS}

Experiment 1: As shown in Table 1, when COCs were treated with Ca-EDTA after at least $24 \mathrm{hr}$ during maturation culture (post-24, post-36 and post-48 groups), more oocytes cleaved parthenogenetically and developed to the blastocyst stage than those in the control and post-12 groups. The cleavage rate of oocytes in the post-36 group was significantly higher $(P<0.05)$ than that of oocytes in the post-24 and post-48 groups; however, there were no significant differences in the rates of blastocyst formation and number of cell per blastocyst among the three groups. In the control and post-12 groups, no oocytes developed to the blastocyst stage.

Experiment 2: When COCs were treated with Ca-EDTA for $36 \mathrm{hr}$ and $48 \mathrm{hr}$ from the start of maturation culture (pre36 and pre-48 groups), the cleavage rate of oocytes gradually increased, and some oocytes developed to the blastocyst stage (Table 2). However, the rates of blastocyst formation were very low to verify significant differences among the groups, irrespective of the Ca-EDTA treatment. In the control, pre-12 and pre-24 groups, no oocytes developed to the blastocyst stage.

As shown in Fig. 2, the PN formation of Ca-EDTA treated oocytes was detected when porcine oocytes were postincubated with Ca-EDTA for at least $12 \mathrm{hr}$ (post-12, post-24, post-36 and post-48). The percentages increased gradually with longer treatment periods, and the rates $(34.2 \%-66.7 \%)$ of PN formation in the post-24, post-36 and post-48 groups were significantly higher $(P<0.05)$ than those $(0 \%)$ in the control group (Fig. 2 A). Furthermore, when COCs were pre-incubated with Ca-EDTA for at least $12 \mathrm{hr}$ (pre-12, pre24 , pre-36 and pre-48), the oocytes formed PN and the rates $(13.4 \%-59.2 \%)$ of PN formation were significantly higher $(P<0.05)$ than those $(0 \%)$ of the control group (Fig. 2B).

\section{DISCUSSION}

In this study, Ca-EDTA treatment during maturation culture not only activated porcine immature oocytes but also induced parthenogenetic development to blastocyst stage. It had been shown that MII-stage oocytes can be parthenogenetically activated by artificial stimuli and that activated oocytes can cleave and develop to the blastocyst stage [12, 13]. Historically, Azuma et al. [3] were the first to report that porcine GV-stage oocytes can be parthenogenetically activated by treatment with $\mathrm{Na}$-EDTA during maturation culture, inducing PN formation, followed by blastocyst formation. Moreover, they demonstrated that Ca-EDTA treatment could also activate porcine oocytes and induce precocious $\mathrm{PN}$ formation during oocyte meiosis $[3,4]$. They suggested that the treatment of porcine oocytes with Ca-EDTA leads to a deficiency of extracellular $\mathrm{Zn}^{2+}$, alters the composition of the membrane and modifies intracellular conditions, eventually resulting in the promotion of parthenogenetic development. Furthermore, they also suggested that Ca-EDTA can promote PN formation not via the MII stage. However, the optimal period for Ca-EDTA treatment during maturation culture to activate oocytes and successful blastocyst formation from $\mathrm{GV}$-stage oocytes remained unclear.

This study demonstrated that when oocytes were treated with Ca-EDTA from $24 \mathrm{hr}$ or $36 \mathrm{hr}$ after the start of maturation culture to the end of the culture period or during the entire IVM (post-24, post-36 and post-48 groups, respectively) and for more than $36 \mathrm{hr}$ from the start of maturation culture (pre-36 and pre-48 groups), oocytes could cleave and develop to the blastocyst stage. These results indicate that Ca-EDTA treatment of at least $24 \mathrm{hr}$ to $36 \mathrm{hr}$ during a maturation culture period of $48 \mathrm{hr}$ is effective in activating porcine immature oocytes to exit meiosis, enter interphase and develop into blastocysts. Also, the oocytes matured for 24 $\mathrm{hr}$ to $36 \mathrm{hr}$ from the start of maturation culture are sensitive to Ca-EDTA activation. In this study, when we performed Ca-EDTA treatment from the start of maturation culture, incubating oocytes with Ca-EDTA for $36 \mathrm{hr}$ was necessary to produce blastocysts. Furthermore, the result of experiment 1 showed that treating oocytes with Ca-EDTA after $24 \mathrm{hr}$ from the start of maturation culture was effective on PN and blastocysts formation. These results indicate that $24 \mathrm{hr}$ to $36 \mathrm{hr}$ from the start of IVM is important period for parthenogenesis of oocytes. In pigs, GVBD typically occurs and metaphase I (MI)-stage oocytes appear between $24 \mathrm{hr}$ and $26 \mathrm{hr}$ after the start of cultivation, and oocytes reach the MII stage after $40 \mathrm{hr}$ to $44 \mathrm{hr}$ of cultivation [2, 24]. Kikuchi et al. [10] reported that in vitro matured porcine oocytes reach to MII stage after $30 \mathrm{hr}$ from the start of maturation culture. We also examined the nuclear status of oocyte matured without Ca-EDTA, and MII-stage oocytes appeared between after 24 $\mathrm{hr}$ to $36 \mathrm{hr}$ from the start of IVM (data not shown). On the other hand, Kim et al. [11] reported the importance of $\mathrm{Zn}^{2+}$ to exit meiosis in mice. $\mathrm{Zn}^{2+}$ content is significantly higher in MII stage oocytes than GV stage oocytes. They also showed that the deficiency of $\mathrm{Zn}^{2+}$ during IVM makes the oocyte retain the telophase I spindle, resulting in the failure to reach 
Table 1. Effects of post-treatment with Ca-EDTA during in vitro maturation culture on the parthenogenetic development of porcine oocytes*

\begin{tabular}{lcccc}
\hline \multirow{2}{*}{ Treatment group** } & \multirow{2}{*}{ No. of oocytes cultured } & \multicolumn{2}{c}{ No. (\%) of embryos } & \multirow{2}{*}{ No. of cells per blastocyst } \\
\cline { 2 - 3 } & & cleaved & developed to blastocyst & \\
\hline Control & 365 & $45(12.5 \pm 2.7)^{\mathrm{a})}$ & $0(0.0)^{\mathrm{a})}$ & - \\
Post-12 & 298 & $23(7.7 \pm 1.6)^{\mathrm{a}}$ & $0(0.0)^{\mathrm{a}}$ & - \\
Post-24 & 300 & $83(27.7 \pm 2.4)^{\mathrm{b})}$ & $10(3.3 \pm 0.7)^{\mathrm{b})}$ & $31.5 \pm 3.7$ \\
Post-36 & 285 & $105(37.0 \pm 5.0)^{\mathrm{c})}$ & $11(4.0 \pm 1.2)^{\mathrm{b}}$ & $29.4 \pm 2.5$ \\
Post-48 & 300 & $81(27.2 \pm 2.6)^{\mathrm{b})}$ & $9(2.6 \pm 0.8)^{\mathrm{b})}$ & $34.7 \pm 3.1$ \\
\hline
\end{tabular}

*All of the experiments were repeated 4 times. Data are expressed as the mean \pm SEM. **Porcine oocytes were cultured without Ca-EDTA from the start of maturation culture and incubated with $1 \mathrm{mM} \mathrm{Ca-EDTA}$ for the designated times until $48 \mathrm{hr}$ of total culture period (Fig. 1). As a control, oocytes were cultured in maturation medium without Ca-EDTA. a-c) Values with different superscript letters in the same column are significantly different $(P<0.05)$.

Table 2. Effects of pre-treatment with Ca-EDTA during in vitro maturation culture on the parthenogenetic development of porcine oocytes*

\begin{tabular}{lcccc}
\hline \multirow{2}{*}{ Treatment group** } & \multirow{2}{*}{ No. of oocytes cultured } & \multicolumn{2}{c}{ No. $(\%)$ of embryos } & \multirow{2}{*}{ No. of cells per blastocyst } \\
\cline { 3 - 4 } & & cleaved & developed to blastocyst & \\
\hline Control & 265 & $\left.21(8.1 \pm 2.9)^{\mathrm{a}}\right)$ & $0(0.0)$ & - \\
Pre-12 & 260 & $24(9.2 \pm 1.3)^{\mathrm{a}}$ & $0(0.0)$ & - \\
Pre-24 & 264 & $28(10.5 \pm 2.9)^{\mathrm{a}}$ & $0(0.0)$ & 34 \\
Pre-36 & 281 & $\left.69(17.8 \pm 3.9)^{\mathrm{a}}, \mathrm{b}\right)$ & $1(0.4 \pm 0.4)$ & 24.5 \\
Pre-48 & 270 & $62(22.7 \pm 4.4)^{\mathrm{b})}$ & $2(0.8 \pm 0.8)$ & \\
\hline
\end{tabular}

*All of the experiments were repeated 4 times. Data are expressed as the mean \pm SEM. **Porcine oocytes were incubated with 1 $\mathrm{mM}$ Ca-EDTA for the designated times from the start of maturation culture and cultured without Ca-EDTA until $48 \mathrm{hr}$ of total culture period (Fig. 1). As a control, oocytes were cultured in maturation medium without Ca-EDTA. a, b) Values with different superscript letters in the same column are significantly different $(P<0.05)$.
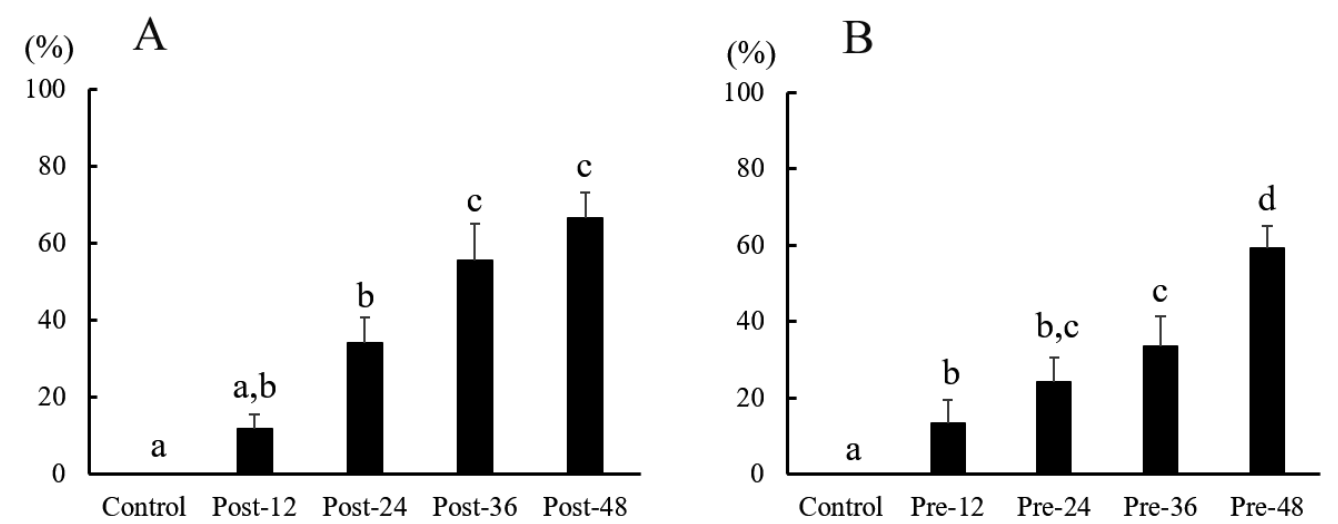

Fig. 2. Effects of post-treatment (A) and pre-treatment (B) with Ca-EDTA during in vitro maturation culture on the pronucleus formation of porcine oocytes after $48 \mathrm{hr}$ of culture (mean \pm SEM). (A) Porcine oocytes were cultured with Ca-EDTA for $0 \mathrm{hr}$ (control), $12 \mathrm{hr}$ (post-12), $24 \mathrm{hr}$ (post-24), $36 \mathrm{hr}$ (post-36) and $48 \mathrm{hr}$ (post-48) after $48 \mathrm{hr}, 36 \mathrm{hr}, 24 \mathrm{hr}, 12 \mathrm{hr}$ and $0 \mathrm{hr}$ from the start of incubation during maturation culture, respectively. (B) Oocytes were cultured with Ca-EDTA for $0 \mathrm{hr}$ (control), $12 \mathrm{hr}$ (pre-12), $24 \mathrm{hr}$ (pre-24), $36 \mathrm{hr}$ (pre-36) and 48 $\mathrm{hr}$ (pre-48) from the start of incubation during maturation culture. Four replicates per treatment group were performed and analyzed. ${ }^{\mathrm{a}-\mathrm{d})}$ Bars with different letters differ significantly $(P<0.05)$.

the MII stage. It is possible that the deficiency of $\mathrm{Zn}^{2+}$ exerts an effect on the release of the polar body (PB) and the PN formed not via the MII stage. Considering these results, the deficiency of $\mathrm{Zn}^{2+}$ by Ca-EDTA treatment during 24 to 36 $\mathrm{hr}$ after the start of maturation culture can be especially effective to oocytes parthenogenesis, which is the period for the transition from the MI to the MII stage. Azuma et al. [4] reported that $77 \%$ of oocytes activated with $1 \mathrm{mM} \mathrm{Ca}$ EDTA for $48 \mathrm{hr}$ formed a PN and $>95 \%$ of the oocytes with PN did not have any PB. In our study, similar rate $(67 \%)$ of PN formation was observed in the oocytes treated with CaEDTA for $48 \mathrm{hr}$ (post- 48 group), but $23 \%$ of the oocytes with 
PN had the PB (data not shown). The difference on the rates of oocytes with the release of $\mathrm{PB}$ is unclear, but our results indicate that some oocytes formed PN after completion of MII. Therefore, further research is necessary to reveal the exact mechanism of the parthenogenetic activation by $\mathrm{Ca}$ EDTA during maturation culture.

In conclusion, porcine immature oocytes can be parthenogenetically activated by Ca-EDTA treatment for at least 24 $\mathrm{hr}$ to $36 \mathrm{hr}$ during maturation culture, leading to $\mathrm{PN}$ formation followed by the formation of blastocysts. Furthermore, oocytes are most sensitive to such treatment at $24 \mathrm{hr}$ to 36 $\mathrm{hr}$ after the start of maturation culture. Our study determined the optimal period of Ca-EDTA treatment required for the parthenogenetic activation of porcine oocytes; however, the underlying mechanism remained unclear. In future studies, we intend to investigate other effectors of porcine parthenogenetic activation to investigate mechanism of chemical activation.

ACKNOWLEDGMENTS. We are grateful to the Meat Inspection Office of Kitakyushu city (Japan) for supplying the porcine ovaries. This work was supported in part by Japan Science and Technology Agency/Japan International Cooperation Agency, Science and Technology Research Partnership for Sustainable Development (JST/JICA, SATREPS).

\section{REFERENCES}

1. Alberio, R., Zakhartchenko, V., Motlik, J. and Wolf, E. 2001. Mammalian oocyte activation: lessons from the sperm and implications for nuclear transfer. Int. J. Dev. Biol. 45: 797-809. [Medline]

2. Anger, M., Klima, J., Kubelka, M., Prochazka, R., Motlik, J. and Schultz, R. M. 2004. Timing of Plk1 and MPF activation during porcine oocyte maturation. Mol. Reprod. Dev. 69: 11-16. [Medline] [CrossRef]

3. Azuma, T., Ikeda, S., Kondo, T., Imai, H. and Yamada, M. 2001. Ethylenediamine-N,N,N',N'-tetraacetic acid induces parthenogenetic activation of porcine oocytes at the germinal vesicle stage, leading to formation of blastocysts. Biol. Reprod. 64: 647-653. [Medline] [CrossRef]

4. Azuma, T., Kondo, T., Ikeda, S., Imai, H. and Yamada, M. 2002. Effects of EDTA saturated with $\mathrm{Ca}^{2+}$ (Ca-EDTA) on pig, bovine and mouse oocytes at the germinal vesicle stage during maturation culture and the involvement of chelation of $\mathrm{Zn}^{2+}$ in pronuclear formation induction by Ca-EDTA. Reproduction 124: 235-240. [Medline] [CrossRef]

5. Campbell, K. H. 1999. Nuclear equivalence, nuclear transfer, and the cell cycle. Cloning 1: 3-15. [Medline] [CrossRef]

6. Collas, P., Fissore, R., Robl, J. M., Sullivan, E. J. and Barnes, F. L. 1993. Electrically induced calcium elevation, activation, and parthenogenetic development of bovine oocytes. Mol. Reprod. Dev. 34: 212-223. [Medline] [CrossRef]

7. Ducibella, T., Huneau, D., Angelichio, E., Xu, Z., Schultz, R. M., Kopf, G. S., Fissore, R., Madoux, S. and Ozil, J. P. 2002. Egg-to-embryo transition is driven by differential responses to $\mathrm{Ca}^{2+}$ oscillation number. Dev. Biol. 250: 280-291. [Medline]
[CrossRef]

8. Heytens, E. 2008. Oocyte activation: The impact of calcium signals on fertilization. Iran. J. Reprod. Med. 6: 171-174.

9. Kawarasaki, T., Otake, M., Tsuchiya, S., Shibata, M., Matsumoto, K. and Isobe, N. 2009. Co-transfer of parthenogenotes and single porcine embryos leads to full-term development of the embryos. Anim. Reprod. Sci. 112: 8-21. [Medline] [CrossRef]

10. Kikuchi, K., Izaike, Y., Noguchi, J., Furukawa, T., Daen, F. P., Naito, K. and Toyoda, Y. 1995. Decrease of histone H1 kinase activity in relation to parthenogenetic activation of pig follicular oocytes matured and aged in vitro. J. Reprod. Fertil. 105: 325-330. [Medline] [CrossRef]

11. Kim, A. M., Vogt, S., O'Halloran, T. V. and Woodruff, T. K. 2010. Zinc availability regulates exit from meiosis in maturing mammalian oocytes. Nat. Chem. Biol. 6: 674-681. [Medline] [CrossRef]

12. Krejkova, T. 2009. Effects of cycloheximide or 6-dimethylaminopurine on the parthenogenetic activation of pig oocytes using pulsatile treatment with nitric oxide donor. Czech J. Anim. Sci. 54: 293-306.

13. Lee, J. W., Tian, X. C. and Yang, X. 2004. Optimization of parthenogenetic activation protocol in porcine. Mol. Reprod. Dev. 68: 51-57. [Medline] [CrossRef]

14. Macháty, Z., Funahashi, H., Mayes, M. A., Day, B. N. and Prather, R. S. 1996. Effects of injecting calcium chloride into in vitro-matured porcine oocytes. Biol. Reprod. 54: 316-322. [Medline] [CrossRef]

15. Mayes, M. A., Stogsdill, P. L. and Prather, R. S. 1995. Parthenogenic activation of pig oocytes by protein kinase inhibition. Biol. Reprod. 53: 270-275. [Medline] [CrossRef]

16. Nagai, T. 1987. Parthenogenetic activation of cattle follicular oocytes in vitro with ethanol. Gamete Res. 16: 243-249. [Medline] [CrossRef]

17. Nagai, T. 1992. Development of bovine in vitro-matured follicular oocytes activated with ethanol. Theriogenology 37: 869-875. [Medline] [CrossRef]

18. Nurse, P. 1990. Universal control mechanism regulating onset of M-phase. Nature 344: 503-508. [Medline] [CrossRef]

19. Nussbaum, D. J. and Prather, R. S. 1995. Differential effects of protein synthesis inhibitors on porcine oocyte activation. Mol. Reprod. Dev. 41: 70-75. [Medline] [CrossRef]

20. Okada, K., Miyano, T. and Miyake, M. 2003. Activation of pig oocytes by intracytoplasmic injection of strontium and barium. Zygote 11: 159-165. [Medline] [CrossRef]

21. Paffoni, A., Brevini, T. A., Gandolfi, F. and Ragni, G. 2008. Parthenogenetic activation: biology and applications in the ART laboratory. Placenta 29 Suppl B: 121-125. [Medline] [CrossRef]

22. Petr, J. 2007. The role of nitric oxide in parthenogenetic activation of pig oocytes: A review. Czech J. Anim. Sci. 52: 363-377.

23. Petters, R. M. and Wells, K. D. 1993. Culture of pig embryos. $J$. Reprod. Fertil. Suppl. 48: 61-73. [Medline]

24. Suzuki, H. and Saito, Y. 2006. Cumulus cells affect distribution and function of the cytoskeleton and organelles in porcine oocytes. Reprod. Med. Biol. 5: 183-194. [CrossRef]

25. Wang, W. H., Macháty, Z., Abeydeera, L. R., Prather, R. S. and Day, B. N. 1998. Parthenogenetic activation of pig oocytes with calcium ionophore and the block to sperm penetration after activation. Biol. Reprod. 58: 1357-1366. [Medline] [CrossRef]

26. Yanagimachi, R. 1988. Mammalian Fertilization. The Physiology of Reproduction 1: 135-185. 